\title{
CONVOLUTION AND RESTRICTION ESTIMATES FOR MEASURES ON CURVES IN $\mathbb{R}^{2}$
}

\author{
DANIEL M. OBERLIN
}

(Communicated by Michael T. Lacey)

\begin{abstract}
We study convolution and Fourier restriction estimates for some degenerate curves in $\mathbb{R}^{2}$.
\end{abstract}

Notational comment: This note concerns certain operators defined on functions on $\mathbb{R}^{2}$. Thus $L^{p}$ will stand for the $L^{p}$ space constructed using Lebesgue measure on $\mathbb{R}^{2}$ and $\|\cdot\|_{p}$ will denote the norm in $L^{p}$. If $E$ is a Borel subset of $\mathbb{R}^{2}$, then $|E|$ will stand for the two-dimensional Lebesgue measure of $E$.

The two following theorems are well known and prototypical for many important results in harmonic analysis.

Theorem 1. Suppose $a<b$ and write $\operatorname{T} f(x)=\int_{a}^{b} f\left(x+\left(t, t^{2}\right)\right) d t$. Then there is a constant $C$ (independent of $a$ and b) such that $\|T f\|_{3} \leq C\|f\|_{3 / 2}$. Also, the collection of points $\left(\frac{1}{p}, \frac{1}{q}\right)$ such that $T$ is bounded from $L^{p}$ into $L^{q}$, the "typeset" of $T$, is the convex hull of the points $(0,0),(1,1)$, and $\left(\frac{2}{3}, \frac{1}{3}\right)$.

Theorem 2. If $1 \leq p<\frac{4}{3}$ and $\frac{1}{p}+\frac{1}{3 q}=1$, there is a constant $C=C(p)$ such that the estimate

$$
\left(\int_{a}^{b}\left|\widehat{f}\left(t, t^{2}\right)\right|^{q} d t\right)^{\frac{1}{q}} \leq C(p)\|f\|_{p}
$$

holds.

It is natural to wonder what happens to Theorems 1 and 2 when the curve $\left(t, t^{2}\right)$ is replaced by a general $(t, \phi(t))$. Since the curvature of the parabola is key to the proofs of Theorems 1 and 2, a reasonable starting point for generalization is the hypothesis $\phi^{\prime \prime} \geq \delta>0$. Furthermore, it has been known for some time that this hypothesis is sufficient to ensure that the analogs of Theorems 1 and 2 hold. The next step is to investigate this situation when $\phi^{\prime \prime}$ is allowed to vanish, and it is easy to see that one can no longer expect the exact analogs of Theorems 1 and 2 to hold. There are then two possibilities. The first is to "dampen" the measure $d t$ by introducing a factor $\omega(t)$ which is small when $\phi^{\prime \prime}(t)$ is small and then to attempt to obtain the exact analogs of Theorems 1 and 2 with $d t$ replaced by $\omega(t) d t$. The second is to keep the $d t$ and to see how the conclusions of Theorems 1 and 2 change.

Concerning the first approach, there are the following results.

Received by the editors October 16, 2006.

2000 Mathematics Subject Classification. Primary 42B10.

Key words and phrases. Convolution, Fourier restriction. 
Theorem 1a $([3])$. Suppose $\phi^{\prime \prime}>0$ and $\phi^{(3)} \geq 0$ on $(a, b)$. Define the operator $T$ by $T f(x)=\int_{a}^{b} f(x+(t, \phi(t))) \phi^{\prime \prime}(t)^{1 / 3} d t$. Then

$$
\left\|T \chi_{E}\right\|_{3} \leq 12^{\frac{1}{3}}\left\|\chi_{E}\right\|_{\frac{3}{2}}
$$

for any measurable $E \subseteq \mathbb{R}^{2}$.

Theorem 2a ([5], [4]). If $1 \leq p<\frac{4}{3}$ and $\frac{1}{p}+\frac{1}{3 q}=1$, there is a constant $C=C(p)$ such that the estimate

$$
\left(\int_{a}^{b}|\widehat{f}(t, \phi(t))|^{q} \phi^{\prime \prime}(t)^{\frac{1}{3}} d t\right)^{\frac{1}{q}} \leq C(p)\|f\|_{p}
$$

holds whenever $\phi$ is as in Theorem $1 a$.

Here are some comments on Theorems 1a and 2a:

(a) The measure $\phi^{\prime \prime}(t)^{1 / 3} d t$ appearing in Theorems 1a and 2a is the so-called affine arclength measure. Its relevance to problems like these was advocated by Drury ([2]). It is the optimal choice of a measure on the graph of $\phi$ for these convolution and restriction problems.

(b) The weakness of Theorem 1a is that the estimate it provides is not a strong type $(3 / 2,3)$ estimate like the one in Theorem 1 , but is (equivalent to) a weak type $(3 / 2,3)$ estimate. Whether the strong type estimate always holds is, to my knowledge, an open problem.

(c) The most interesting feature of Theorems 1a and 2a is that the estimates they contain are uniform in the sense that they are independent both of $\phi$ (subject to the monotonicity hypotheses imposed on $\phi$ ) and of the length of the interval $(a, b)$.

The purpose of this note, however, is to explore the second-mentioned approach above (keep the $d t$ ) and, in particular, to prove Theorems $1 \mathrm{~b}$ and $2 \mathrm{~b}$ below. In what follows, $d \lambda$ will refer to the measure on $\mathbb{R}^{2}$ corresponding to $d t$ on the graph $\gamma$ of $\phi$. We will be interested in estimates on $\lambda$ of the form

$$
\lambda(P) \leq c|P|^{\alpha}
$$

to hold for all parallelograms $P \subset \mathbb{R}^{2}$ and where $0<\alpha \leq 1 / 3$.

Theorem 1b. Suppose that $\phi:(a, b) \rightarrow \mathbb{R}$ is a convex $C^{(1)}$ function. Define the operator $T$ by $T f(x)=\int_{a}^{b} f(x+(t, \phi(t))) d t$ and assume that (2) holds. Then the estimate

$$
\|T f\|_{q} \leq C(p, q) c\|f\|_{p}
$$

holds for $\left(\frac{1}{p}, \frac{1}{q}\right)=(2 \alpha, \alpha)$ whenever $f=\chi_{E}$ and $E$ is a Borel subset of $\mathbb{R}^{2}$. On the other hand, if $\frac{1}{p}-\frac{1}{q}=\alpha$ and if (3) holds (for $f=\chi_{E}$ ) without the $C(p, q)$, then (2) holds with $c$ replaced by $\widetilde{C}(p) \cdot c$.

Theorem 2b. With $\phi$ as in Theorem 1b, suppose that (2) holds. Then the estimate

$$
\left(\int_{a}^{b}|\widehat{f}(t, \phi(t))|^{q} d t\right)^{\frac{1}{q}} \leq c^{\frac{1}{q}} \cdot C(p)\|f\|_{p}
$$

holds whenever $\frac{1}{q}=\frac{1}{\alpha}\left(1-\frac{1}{p}\right)$ and $1 \leq p<\frac{2-2 \alpha}{2-3 \alpha}$. If, on the other hand, (4) holds without the $C(p)$ for some $p$ and $q$ satisfying $\frac{1}{q}=\frac{1}{\alpha}\left(1-\frac{1}{p}\right)$, then $(2)$ holds with $c$ replaced by $\widetilde{C}(p) \cdot c$. 
Here are some remarks on these results:

(d) If an estimate (3) is to hold even for $f=\chi_{E}$, then the point $(1 / p, 1 / q)$ must lie in the triangle $\mathcal{T}$ which is the closed convex hull of the points $(0,0),(1,1)$, and $(2 / 3,1 / 3)$. This is basically a consequence of the fact that the dimension of the graph of $\phi$ is one. The point $(1 / p, 1 / q)=(2 \alpha, \alpha)$ is a point of intersection of the line $1 / p-1 / q=\alpha$ with the boundary of this triangle. If (3) holds for $f=\chi_{E}$ when $(1 / p, 1 / q)=(2 \alpha, \alpha)$ and if $\alpha<1 / 3$, then standard arguments show that (3) must hold whenever $1 / p-1 / q=\alpha$ and $(1 / p, 1 / q)$ is in the interior of the triangle $\mathcal{T}$. Thus the following are equivalent: $(2)$; (3) for $f=\chi_{E}$ and $(1 / p, 1 / q)=(2 \alpha, \alpha)$; (3) for all $(1 / p, 1 / q)$ in the interior of $\mathcal{T}$ with $1 / p-1 / q=\alpha$; (4) for any $(1 / p, 1 / q)$ with $1 / q=(1-1 / p) / \alpha$ and $p>1$; and (4) for all $(1 / p, 1 / q)$ with $1 / q=(1-1 / p) / \alpha$ with $1 \leq p<(2-2 \alpha) /(2-3 \alpha)$. The first three of these equivalences can be viewed as a partial response to an old problem of Stein [6], pp. 122-123: "characterize (if possible, in terms of the size of the measure $d \mu$, whatever that means) the condition of $f \rightarrow f * d \mu$ yielding a bounded operator from an $L^{p}$ space to an $L^{r}$ space".

(e) Theorem $1 \mathrm{~b}$ is sharp up to the fact that (3) is proven only for $f=\chi_{E}$.

(f) Although the relation $1 / q=(1-1 / p) / \alpha$ in Theorem $2 \mathrm{~b}$ is also sharp, the range $1 \leq p<(2-2 \alpha) /(2-3 \alpha)$ is not best possible if $\alpha<1 / 3$ - see Theorem 1.3 in $[1]$.

The remainder of this note consists of the proofs of Theorems $1 \mathrm{~b}$ and $2 \mathrm{~b}$.

Proof of Theorem $1 b$. Suppose that $\phi$ is a convex $C^{(1)}$ function defined on $(a, b)$ for which (2) holds and assume (for the moment) the following result.

Lemma. If (2) holds and $A$ is a Borel subset of $(a, b)$, then

$$
m_{1}(A)^{\frac{1}{\alpha}-1} \leq c^{\frac{1}{\alpha}} 2^{1+\frac{1}{\alpha}} \int_{a}^{b} \chi_{A}(t)\left|\phi^{\prime}(t)-\phi^{\prime}(s)\right| d t
$$

for any $s \in(a, b)$.

We modify the proof from [3]:

$$
\int_{\mathbb{R}^{2}}\left(T \chi_{E}(x)\right)^{\frac{1}{\alpha}} d x=\int_{\mathbb{R}^{2}} \chi_{E}(x) \int_{a}^{b}\left(\int_{a}^{b} \chi_{E}(x+(t-s, \phi(t)-\phi(s))) d t\right)^{\frac{1}{\alpha}-1} d s d x .
$$

Applying the lemma to the inside integral,

$$
\begin{gathered}
\int_{a}^{b}\left(\int_{a}^{b} \chi_{E}(x+(t-s, \phi(t)-\phi(s))) d t\right)^{\frac{1}{\alpha}-1} d s \\
\leq c^{\frac{1}{\alpha}} 2^{1+\frac{1}{\alpha}} \int_{a}^{b} \int_{a}^{b} \chi_{E}(x+(t-s, \phi(t)-\phi(s)))\left|\phi^{\prime}(t)-\phi^{\prime}(s)\right| d t d s .
\end{gathered}
$$

The fact that the Jacobian of the map

$$
(t, s) \rightarrow(t-s, \phi(t)-\phi(s))
$$

is $\left|\phi^{\prime}(t)-\phi^{\prime}(s)\right|$ yields

$$
\int_{a}^{b} \int_{a}^{b} \chi_{E}(x+(t-s, \phi(t)-\phi(s)))\left|\phi^{\prime}(t)-\phi^{\prime}(s)\right| d t d s \leq m_{2}(E),
$$

and it follows that

$$
\int_{a}^{b}\left(\int_{a}^{b} \chi_{E}(x+(t-s, \phi(t)-\phi(s))) d t\right)^{\frac{1}{\alpha}-1} d s \leq c^{\frac{1}{\alpha}} 2^{1+\frac{1}{\alpha}} m_{2}(E) .
$$


Thus from (6),

$$
\int_{\mathbb{R}^{2}}\left(T \chi_{E}(x)\right)^{\frac{1}{\alpha}} d x \leq c^{\frac{1}{\alpha}} 2^{1+\frac{1}{\alpha}} m_{2}(E)^{2}
$$

as desired.

The reverse implication of Theorem $1 \mathrm{~b}$ follows easily by taking $f=\chi_{P+P}$ in (3). Thus the proof of Theorem $1 \mathrm{~b}$ will be complete after the proof of the lemma.

Proof of the lemma. Let $I$ be an interval such that

$$
m_{1}(I)=m_{1}(A), s \in I \subseteq(a, b),
$$

and

$$
\int_{I}\left|\phi^{\prime}(t)-\phi^{\prime}(s)\right| d t \leq \int_{A}\left|\phi^{\prime}(t)-\phi^{\prime}(s)\right| d t
$$

(To see that $I$ exists, let $J(t, s)$ be an interval with endpoints $t$ and $s$, define $\tau: A \rightarrow(a, b)$ by $\tau(t)=s \pm m_{1}(A \cap J(t, s))$ with the choice of + or - depending on whether $t>s$ or $t<s$. Note that $\tau$ is a measure-preserving map of $A$ onto some interval $I$ containing $s$, and observe that $|\tau(t)-s| \leq|t-s|$ implies that $\left|\phi^{\prime}(\tau(t))-\phi^{\prime}(s)\right| \leq\left|\phi^{\prime}(t)-\phi^{\prime}(s)\right|$.) Suppose that $\widetilde{a}$ and $\widetilde{b}$ are the endpoints of $I$. Then either $\widetilde{b}-s \geq m_{1}(A) / 2$ or $s-\widetilde{a} \geq m_{1}(A) / 2$. In the first case let $P$ be the parallelogram with one side the tangent to the graph of $\phi$ at $(s, \phi(s))$, with one side vertical through $(\widetilde{b}, \phi(\widetilde{b}))$, and with the points $(s, \phi(s)),(\widetilde{b}, \phi(\widetilde{b}))$ as two of its vertices. Then

$$
\begin{aligned}
\frac{m_{2}(P)}{2} & \leq \int_{s}^{\widetilde{b}}\left(\phi(\widetilde{b})-\phi^{\prime}(s)(\widetilde{b}-v)-\phi(v)\right) d v=\int_{s}^{\widetilde{b}} \int_{v}^{\widetilde{b}}\left(\phi^{\prime}(t)-\phi^{\prime}(s)\right) d t d v \\
& =\int_{s}^{\widetilde{b}}\left(\phi^{\prime}(t)-\phi^{\prime}(s)\right)(t-s) d t \leq m_{1}(A) \int_{\widetilde{a}}^{\widetilde{b}}\left|\phi^{\prime}(t)-\phi^{\prime}(s)\right| d t .
\end{aligned}
$$

That is, recalling the definition of $\lambda$ and then (2),

$$
\left(\frac{m_{1}(A)}{2 c}\right)^{\frac{1}{\alpha}} \leq\left(\frac{\widetilde{b}-s}{c}\right)^{\frac{1}{\alpha}}=\left(\frac{\lambda(P)}{c}\right)^{\frac{1}{\alpha}} \leq m_{2}(P) \leq 2 m_{1}(A) \int_{\widetilde{a}}^{\widetilde{b}}\left|\phi^{\prime}(t)-\phi^{\prime}(s)\right| d t .
$$

Thus

$$
\frac{m_{1}(A)^{\frac{1}{\alpha}-1}}{(2 c)^{\frac{1}{\alpha}}} \leq 2 \int_{A}\left|\phi^{\prime}(t)-\phi^{\prime}(s)\right| d t,
$$

yielding (5) in the case $\widetilde{b}-s \geq m_{1}(A) / 2$. The proof if $s-\widetilde{a} \geq m_{1}(A) / 2$ is analogous.

Proof of Theorem 2b. First assume that (2) holds. Then (7) is true as well, and, with (7), the proof of (4) is a simple adaptation of the proof of the theorem in [4].

Recalling that $d \lambda$ is $d t$ on the graph of $\phi$. We will establish the estimate

$$
\left\|\widehat{\chi_{\gamma(F)} d} \lambda\right\|_{L^{s, \infty}\left(\mathbb{R}^{2}\right)} \leq C(c, r) m_{1}(F)^{\frac{1}{r}} \quad \text { if } 1-\frac{1}{r}=\frac{1}{\alpha s}, 1 \leq r<\frac{2-2 \alpha}{1-2 \alpha},
$$

and $F \subseteq(a, b)$. Since $r \leq s$, the Marcinkiewicz interpolation theorem and then a duality argument will yield Theorem $2 \mathrm{~b}$.

If $1 / d+2 / s=1$ or $1 / d=1-2 \alpha+2 \alpha / r$ (which are equivalent given $1-1 / r=$ $1 /(\alpha s))$, we will actually show that

$$
\left\|\left(\chi_{\gamma(F)} d \lambda\right) *\left(\chi_{\gamma(F)} d \lambda\right)^{\sim}\right\|_{L^{d, \infty}\left(\mathbb{R}^{2}\right)} \leq C(c, r) m_{1}(F)^{\frac{2}{r}}
$$


whenever $F$ is a Borel subset of $(a, b)$. Then it will follow that

$$
\begin{aligned}
& \left\|\left(\widehat{\chi_{\gamma(F)} d} \lambda\right)^{2}\right\|_{L^{\frac{s}{2}, \infty}\left(\mathbb{R}^{2}\right)}^{\frac{1}{2}} \\
& \quad \leq C(c, r)\left\|\left(\chi_{\gamma(F)} d \lambda\right) *\left(\chi_{\gamma(F)} d \lambda\right)^{\sim}\right\|_{L^{d, \infty}\left(\mathbb{R}^{2}\right)}^{\frac{1}{2}} \leq C(c, r) m_{1}(F)^{\frac{1}{r}}
\end{aligned}
$$

by Hunt's generalization of the Hausdorff-Young theorem, and this is (8). Now (9) is true for all $(r, d)$ of interest if it is true for the two extreme cases $(r, d)=(1,1)$ and $(r, d)=((2-2 \alpha) /(1-2 \alpha),(1-\alpha) /(1-2 \alpha))$. The first of these is easy and so it is enough to establish the inequality

$$
\left\|\left(\chi_{\gamma(F)} d \lambda\right) *\left(\chi_{\gamma(F)} d \lambda\right)^{\sim}\right\|_{L^{\frac{1-\alpha}{1-2 \alpha}, \infty}\left(\mathbb{R}^{2}\right)} \leq C(c, \alpha) m_{1}(F)^{\frac{1-2 \alpha}{1-\alpha}} .
$$

Inequality (10) is a consequence of the estimate

$$
\begin{gathered}
\int_{a}^{b} \int_{a}^{b} \chi_{E}(t-u, \phi(t)-\phi(u)) \chi_{F}(u) \chi_{F}(t) d u d t \\
\leq C(c, \alpha) m_{1}(F)^{\frac{1-2 \alpha}{1-\alpha}} m_{2}(E)^{\frac{\alpha}{1-\alpha}}
\end{gathered}
$$

for Borel subsets $E$ of $\mathbb{R}^{2}$. But the left-hand side of (11) is bounded by

$$
\left(\int_{a}^{b}\left(\int_{a}^{b} \chi_{E}(t-u, \phi(t)-\phi(u)) d u\right)^{\frac{1-\alpha}{\alpha}} d t\right)^{\frac{\alpha}{1-\alpha}} m_{1}(F)^{\frac{1-2 \alpha}{1-\alpha}} .
$$

Thus (11) follows from (7), proving (8) and so (4).

If, on the other hand, (4) holds for $p, q$ with $1 / q=(1-1 / p) / \alpha$, then (2) follows from the (easily verified) existence of $f$ with $\chi_{P} \leq \widehat{f}$ and $\|f\|_{p} \leq C(p) m_{2}(P)^{1-1 / p}$. This completes the proof of Theorem $2 \mathrm{~b}$.

\section{ACKNOWLEDGMENTS}

The author would like to thank J.-G. Bak and Andreas Seeger for (electronic) conversations concerning Theorem $2 \mathrm{~b}$. The author is also grateful to an anonymous referee for several comments and corrections.

\section{REFERENCES}

[1] J.-G. Bak, D. Oberlin, and A. Seeger, Restriction of Fourier transforms to degenerate simple curves, J. Australian Math. Soc., to appear.

[2] S. W. Drury, Degenerate curves and harmonic analysis, Math. Proc. Camb. Phil. Soc. 108 (1990), 89-96. MR1049762 (91h:42021)

[3] D. Oberlin, Convolution with affine arclength measures in the plane, Proc. Amer. Math. Soc. 127 (1999), 3591-3592. MR1690999 (2000c:42016)

[4] D. Oberlin, Fourier restriction for affine arclength measures in the plane, Proc. Amer. Math. Soc. 129 (2001), 3303-3305. MR1845006 (2002g:42013)

[5] P. Sjölin, Fourier multipliers and estimates of the Fourier transform of measures carrried by smooth curves in $\mathbb{R}^{2}$, Studia Math. 51 (1974), 169-182. MR0385437 (52:6299)

[6] E. M. Stein, Harmonic analysis in $\mathbb{R}^{n}$, Studies in Harmonic Analysis, MAA Studies in Mathematics 13, Mathematical Association of America, Washington, D.C., 1976. MR0461002 $(57: 990)$ 4510

Department of Mathematics, Florida State University, Tallahassee, Florida 32306-

E-mail address: oberlin@math.fsu.edu 